\title{
Treatment Patterns, Complications, and Health Care Utilization Among Endometriosis Patients Undergoing a Laparoscopy or a Hysterectomy: A Retrospective Claims Analysis
}

Eric S. Surrey · Ahmed M. Soliman · Hongbo Yang · Ella Xiaoyan Du •

Bowdoin $\mathrm{Su}$

Received: June 8, 2017 / Published online: October 16, 2017

(c) The Author(s) 2017. This article is an open access publication

\section{ABSTRACT}

Introduction: Hysterectomy and laparoscopy are common surgical procedures used for the treatment of endometriosis. This study compares outcomes for women who received either procedure within the first year post initial surgery.

Methods: The study used data from the Truven Health MarketScan claims databases from 2004 to 2013 to identify women aged $18-49$ years who received an endometriosis-related

Enhanced content To view enhanced content for this article go to http://www.medengine.com/Redeem/ 496CF060409A7267.

Electronic supplementary material The online version of this article (doi:10.1007/s12325-017-0619-3) contains supplementary material, which is available to authorized users.

E. S. Surrey

Colorado Center for Reproductive Medicine, Lone

Tree, CO 80124, USA

A. M. Soliman · B. Su

AbbVie, Inc., 1 North Waukegan Road, North

Chicago, IL 60064, USA

H. Yang ( $\square)$

Analysis Group, Inc., 111 Huntington Avenue,

Boston, MA 02199, USA

e-mail: hongbo.yang@analysisgroup.com

E. X. Du

Analysis Group, Inc., 335 S. Hope Street, 27th Floor,

Los Angeles, CA 90071, USA laparoscopy or hysterectomy. Patients were excluded if they did not have continuous insurance coverage from 1 year before through 1 year after their endometriosis-related procedure, if they were diagnosed with uterine fibroids prior to or on the date of surgery (i.e., index date), or if they had a hysterectomy prior to the index date. The descriptive analyses examined differences between patients with an endometriosis-related laparoscopy or hysterectomy in regard to medications prescribed, complications, and hospitalizations during the immediate year post procedure.

Results: The final sample consisted of 24,915 women who underwent a hysterectomy and 37,308 who underwent a laparoscopy. Results revealed significant differences between the cohorts, with women who received a laparoscopy more likely to be prescribed a GnRH agonist, progestin, danazol, or an opioid analgesic in the immediate year post procedure compared to women who underwent a hysterectomy. In contrast, women who underwent a hysterectomy generally had higher complication rates. Index hospitalization rates and length of stay (LOS) were higher for women who had a hysterectomy, while post-index hospitalization rates and LOS were higher for women who had a laparoscopy. For both cohorts, post-procedure complications were associated with significantly higher hospitalization rates and longer LOS. 
Conclusion: This study indicated significantly different 1-year post-surgical outcomes for patients who underwent an endometriosis-related hysterectomy relative to a laparoscopy. Furthermore, the endometriosis patients in this analysis had a considerable risk of surgical complications, subsequent surgeries, and hospital admissions, both during and after their initial therapeutic laparoscopy or hysterectomy. Funding: AbbVie.

Keywords: Endometriosis; Health care utilization; Hysterectomy; Laparoscopy; Treatment patterns

\section{INTRODUCTION}

In the USA, up to $10 \%$ of women of reproductive age, $50 \%$ of women with infertility, and $70-90 \%$ of women with pelvic pain have endometriosis, characterized by the presence of uterine lining (endometrium) outside the uterus $[1,2]$. The exact prevalence of the disease is unclear, since endometriosis can be asymptomatic and is likely underdiagnosed $[3,4]$. The most common symptom of endometriosis is abdominal/pelvic pain; other symptoms include but are not limited to abnormal bleeding, digestive issues, fatigue, and infertility $[3,5]$. A multi-country costs-of-illness study reported that the economic burden associated with endometriosis is significant and comparable to that of diabetes, Crohn's disease, or rheumatoid arthritis [6]. In the USA, the direct costs of endometriosis per patient, per year have been estimated at $\$ 12,118$ [7] with hospitalizations reported to be the largest driver of these costs [8].

In the absence of a cure, endometriosis treatments aim to manage symptoms, especially pain and infertility, and include drug therapies (analgesic and hormone) and surgery [1, 2]. While drug therapies have been shown to reduce pain $[9,10]$, surgery is currently the only treatment option shown to improve fertility rates [11], and it also may be used to treat pain that is unresponsive to pharmacologic agents alone $[12,13]$. Laparoscopy is the most conservative surgical approach, and in mild to moderate disease it is the type of surgery most often used [14] to remove endometriosis-related lesions and to relieve pain $[9,15]$. Alternatively, in certain severe cases, endometriosis may be treated through the complete or partial removal of the uterus (hysterectomy), possibly in combination with the removal of one or both ovaries (oophorectomy) and the fallopian tubes (salpingectomy) [7, 12]. In general, however, treatment for endometriosis-related pain has been challenging for medical practitioners and often delayed and suboptimal for patients [1-3]. Laparoscopy has been associated with a substantial rate of disease recurrence (30-50\%) within 5 years $[16,17]$ and a repeat surgery rate as high as $55 \%$ within 7 years [18]. Previous evidence of hysterectomy outcomes has been inconsistent, leaving physicians and patients to wonder, for instance, whether it is best to preserve the ovaries during the hysterectomy, as some have argued [13, 19], or to remove both ovaries along with the uterus $[16,18]$. As a result, the American Society for Reproductive Medicine has argued that there is a need for further research examining the benefits and costs of the current endometriosis treatment strategies [1].

To this end, the present study retrospectively observed the therapeutic patterns and outcomes of women with endometriosis who received a laparoscopy or a hysterectomy, as reported in a large national US insurance claims database. Extending for a 12-month period after the index surgery, this descriptive analysis compared postoperative treatments, complications, hospitalization rates, and hospital length of stay (LOS) experienced by the two cohorts of patients.

\section{METHODS}

Data for this descriptive, naturalistic cohort study were obtained from the Truven Health Analytics Marketscan Commercial Claims and Encounters (CCAE) database. The CCAE database contains retrospective claims information on patient demographics, enrollment, inpatient, outpatient, and prescription drug use from a geographically diverse population living 
across the USA. The data used in this study spanned the time period from January 1, 2004 through December 31, 2013 and complied fully with the Health Insurance Portability and Accountability Act (HIPAA). The article does not contain any new studies with human or animal subjects performed by any of the authors.

Data for this analysis was used to estimate retreatment rates of patients who underwent a laparoscopy or hysterectomy, with a focus on surgical rates [20]. Consistent with the prior research, to be included in this study women aged 18-49 years had to have received a diagnosis of endometriosis (International Classification of Diseases Ninth Revision [ICD-9] code of 617.xx) between 2004 and 2013 and to have undergone at least one endometriosis-related laparoscopy or hysterectomy on or after the earliest diagnosis date for endometriosis, with such a procedure date identified as the index date. Endometriosis-related laparoscopies and hysterectomies were defined as the receipt of a procedure code for a laparoscopy or a hysterectomy with an accompanying diagnosis of endometriosis. Finally, patients were also required to have continuous insurance coverage from at least 1 year before through at least 1 year after the index date. Exclusion criteria included the receipt of a diagnosis of uterine fibroids (ICD-9 of 218.xx) before or on the index date or a hysterectomy before the index date. Figure 1 illustrates how each of the inclusion and exclusion criteria affected the sample size.

Given the above inclusion and exclusion criteria, the 62,223 women included in the study were classified as hysterectomy $(N=24,915)$ or laparoscopy $(N=37,308)$ patients according to the initial surgical procedure, with individuals who received both such procedures $(N=1956)$ classified as hysterectomy patients. In addition to examining baseline characteristics, the study compared the 1-year post-index outcomes of the laparoscopy and hysterectomy cohorts. Specifically, the analyses examined all of the treatments, complications, and hospitalizations that occurred during the first year after the index surgery. The observed treatments included combined oral contraceptives and nonsteroidal anti-inflammatory drugs (NSAIDSs), opioid and
Female with at least one diagnosis of endometriosis $\mathbf{N}=717,941$

At least 1 endometriosis-related hysterectomy or laparoscopy on or after endometriosis diagnosis (index date) and no hysterectomy prior to index date

$\mathrm{N}=405,991$
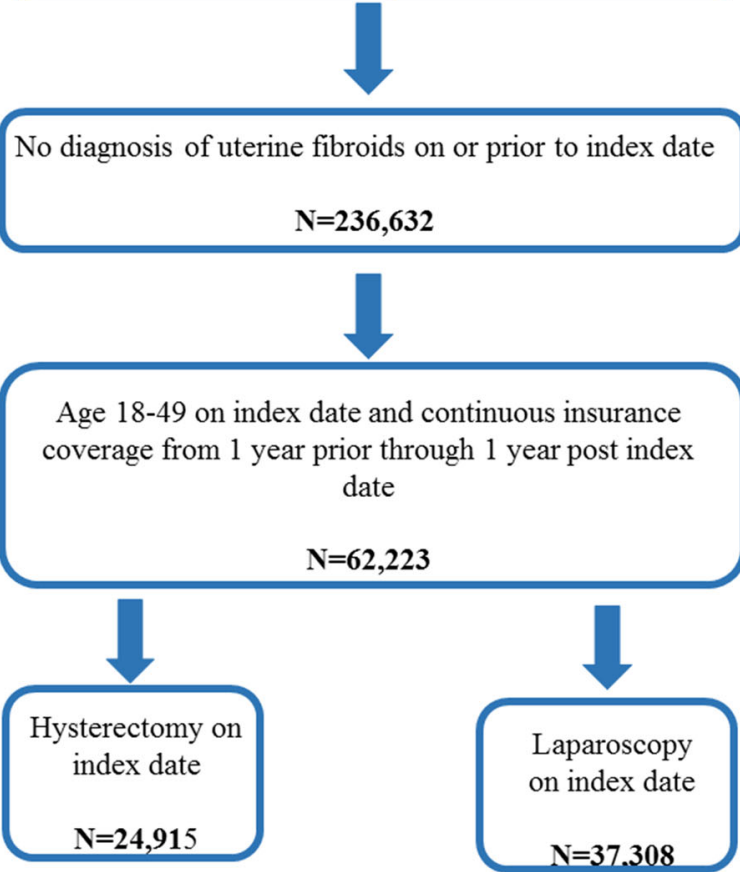

Fig. 1 Inclusion-exclusion criteria and sample size. Patients with both a hysterectomy and a therapeutic laparoscopy on the index date were assigned to the hysterectomy cohort. There were 1956 patients with both procedures on the index date

non-opioid analgesics, gonadotropin-releasing hormone (GnRH) agonists, progestins, and danazol, which is a synthetic steroid used in the management of endometriosis. The observed complications included venous thromboembolism, nephropathy, infection, menopausal symptoms, vascular repair, damage to blood vessels, and bleeding. Other complications examined were gastritis and duodenitis, genitourinary tract injuries, gastrointestinal tract injuries, nerve injuries, vaginal cuff dehiscence, and repairs to the bladder, ureter, or bowel. 
Endometriosis-related surgeries performed after the initial procedure were also examined and included hysterectomy, therapeutic laparoscopy, oophorectomy, laparotomy, and fallopian tube removal (salpingectomy). The investigation also included a comparison of hospitalization rates associated with index surgery or during the 1 year post procedure (all-cause and endometriosis-related), and LOS based upon index procedure (hysterectomy or laparoscopy) and upon complication status. The all-cause and endometriosis-related hospitalization rates by 14 days, 1 and 3 months, respectively, were also described and compared between the two cohorts.

The analysis was primarily descriptive in nature, and descriptive statistics (mean and standard deviation for continuous variables, and frequency and percentages for categorical variables) were used to characterize the sample. Wilcoxon rank sum tests and Chi square tests were utilized to assess differences between the hysterectomy and laparoscopy cohorts. All analyses were conducted using SAS, version 9.3 (SAS Institute, Inc., Cary, NC). A $P$ value less than 0.05 was considered to be statistically significant.

\section{RESULTS}

The results revealed significant differences in the characteristics, treatments, and diagnoses of the two cohorts prior to their index surgeries (see Table 1). For instance, hysterectomy patients were older than the laparoscopy cohort at baseline (mean of 41.4 years, $S D=5.2$ years vs mean of 35.4 years, $\mathrm{SD}=7.5$ years; $P<0.0001$ ). In addition, there were regional differences between the two cohorts $(P<0.0001)$, with hysterectomy patients, compared to laparoscopy patients, more likely to reside in the South (54.0\% vs $48.2 \%)$ and less likely to reside in the Northeast $(6.7 \%$ vs $9.9 \%)$. There were also statistically significant differences between the two cohorts with regards to insurance type $(P<0.0001)$, with women who had a hysterectomy more likely to be insured via a preferred provider organization $(65.2 \%$ vs $64.0 \%)$.

Table 2 reveals the medical treatments provided at 3, 6 months, and 1 year after the initial procedure. At all three time horizons, the women who received a laparoscopy were more likely than those who received a hysterectomy to be prescribed a combined oral contraceptive, a GnRH agonist, progestin, or danazol. In contrast, the women who underwent a hysterectomy were more likely to have been prescribed an NSAID. Women who underwent a hysterectomy were also more likely to have received a prescription for a combined oral contraceptive and NSAID at 3 or 6 months post index procedure but less likely to have received such a prescription at 1 year post index procedure. Table 2 also shows that the proportion of patients who were prescribed a medical treatment tended to grow over time. For example, the use of non-opioid analgesics approximately doubled from 6 to 12 months post procedure, while the use of GnRH agonists and progestin increased over $50 \%$ from 3 to 12 months post procedure.

Table 3 compares 1-year post-procedure complications between hysterectomy and laparoscopy patients. Results illustrate that nearly half $(46.0 \%)$ of patients who underwent a hysterectomy and over one-third (36.4\%) of patients who underwent a laparoscopy experienced at least one complication, with patients in both cohorts most commonly identified with medical/surgical complications, menopausal symptoms, gastritis and duodenitis, and vascular repair. Hysterectomy patients were significantly more likely to experience complications compared to the laparoscopy cohort. Specifically, the hysterectomy patients were more likely to be diagnosed with medical/surgical complications, venous thromboembolism, menopausal symptoms, nephropathy, infection, genitourinary tract infection, bleeding, vaginal cuff dehiscence, nerve injury, repair to ureter, repair to bowel, or other complications (accidental cuts, puncture, perforations, or hemorrhage during medical care and surgical procedures). In contrast, the laparoscopy patients were significantly more likely to be diagnosed with a repair to the bladder in the year after the index date. There was no statistically significant difference between the two cohorts with regards to post-index diagnoses of gastritis and duodenitis, vascular repair, damage 
Table 1 Baseline characteristics for endometriosis patients who received hysterectomy or laparoscopy

\begin{tabular}{|c|c|c|}
\hline \multirow[t]{2}{*}{ Baseline characteristic $^{a}$} & \multicolumn{2}{|l|}{ Endometriosis patients* } \\
\hline & With hysterectomy $N=24,915$ & With laparoscopy $N=37,308$ \\
\hline Age, mean (SD) & $41.4(5.2)$ & $35.4(7.5)$ \\
\hline $18-34$ & $2921(11.7 \%)$ & $16,340(43.8 \%)$ \\
\hline $35-49$ & $21,994(88.3 \%)$ & $20,968(56.2 \%)$ \\
\hline \multicolumn{3}{|l|}{ Region, $n$ (\%) } \\
\hline Northeast & $1658(6.7 \%)$ & $3680(9.9 \%)$ \\
\hline North Central & $5873(23.6 \%)$ & $9987(26.8 \%)$ \\
\hline South & $13,458(54.0 \%)$ & $17,977(48.2 \%)$ \\
\hline West & $3554(14.3 \%)$ & $5175(13.9 \%)$ \\
\hline Unspecified & $372(1.5 \%)$ & $489(1.3 \%)$ \\
\hline \multicolumn{3}{|l|}{ Insurance type, $n(\%)$} \\
\hline Comprehensive & $305(1.2 \%)$ & $637(1.7 \%)$ \\
\hline Health maintenance organization (HMO) & $3430(13.8 \%)$ & $5352(14.3 \%)$ \\
\hline Non-capitated point-of-service & $2189(8.8 \%)$ & $3411(9.1 \%)$ \\
\hline Preferred provider organization (PPO) & $16,243(65.2 \%)$ & $23,891(64.0 \%)$ \\
\hline Other health plan & $2748(11.0 \%)$ & $4017(10.8 \%)$ \\
\hline \multicolumn{3}{|l|}{ Year of index date, $n(\%)$} \\
\hline $2004-2005^{\mathrm{b}}$ & $1737(7.0 \%)$ & $2866(7.7 \%)$ \\
\hline 2006 & $1638(6.6 \%)$ & $2576(6.9 \%)$ \\
\hline 2007 & $1753(7.0 \%)$ & $2712(7.3 \%)$ \\
\hline 2008 & $3253(13.1 \%)$ & $4655(12.5 \%)$ \\
\hline 2009 & $3475(13.9 \%)$ & $5004(13.4 \%)$ \\
\hline 2010 & $3567(14.3 \%)$ & $5397(14.5 \%)$ \\
\hline 2011 & $4872(19.6 \%)$ & $7387(19.8 \%)$ \\
\hline 2012 & $4620(18.5 \%)$ & $6711(18.0 \%)$ \\
\hline
\end{tabular}

${ }^{*}$ All differences between hysterectomy and laparoscopy cohorts were statistically significant on the basis of Wilcoxon rank-sum tests for continuous variables and Chi square tests for categorical variables $(P<0.05)$

${ }^{a}$ Baseline characteristics were assessed on the index date

b Included 3 patients with an index date on December 31, 2004

to blood vessels, or injury to gastrointestinal tract.

Table 4 compares hospitalization rates and LOS among patients who initially received a hysterectomy or a laparoscopy, while also examining differences in hospitalization rates and LOS based upon complication status. Results from Table 4 reveal that a hysterectomy was associated with a higher rate of hospitalization $(58.4 \%$ vs $2.9 \% ; P<0.0001)$ and a longer LOS ( 1.8 vs 0.1 days; $P<0.0001)$ at the time of the index procedure. Meanwhile, a laparoscopy 


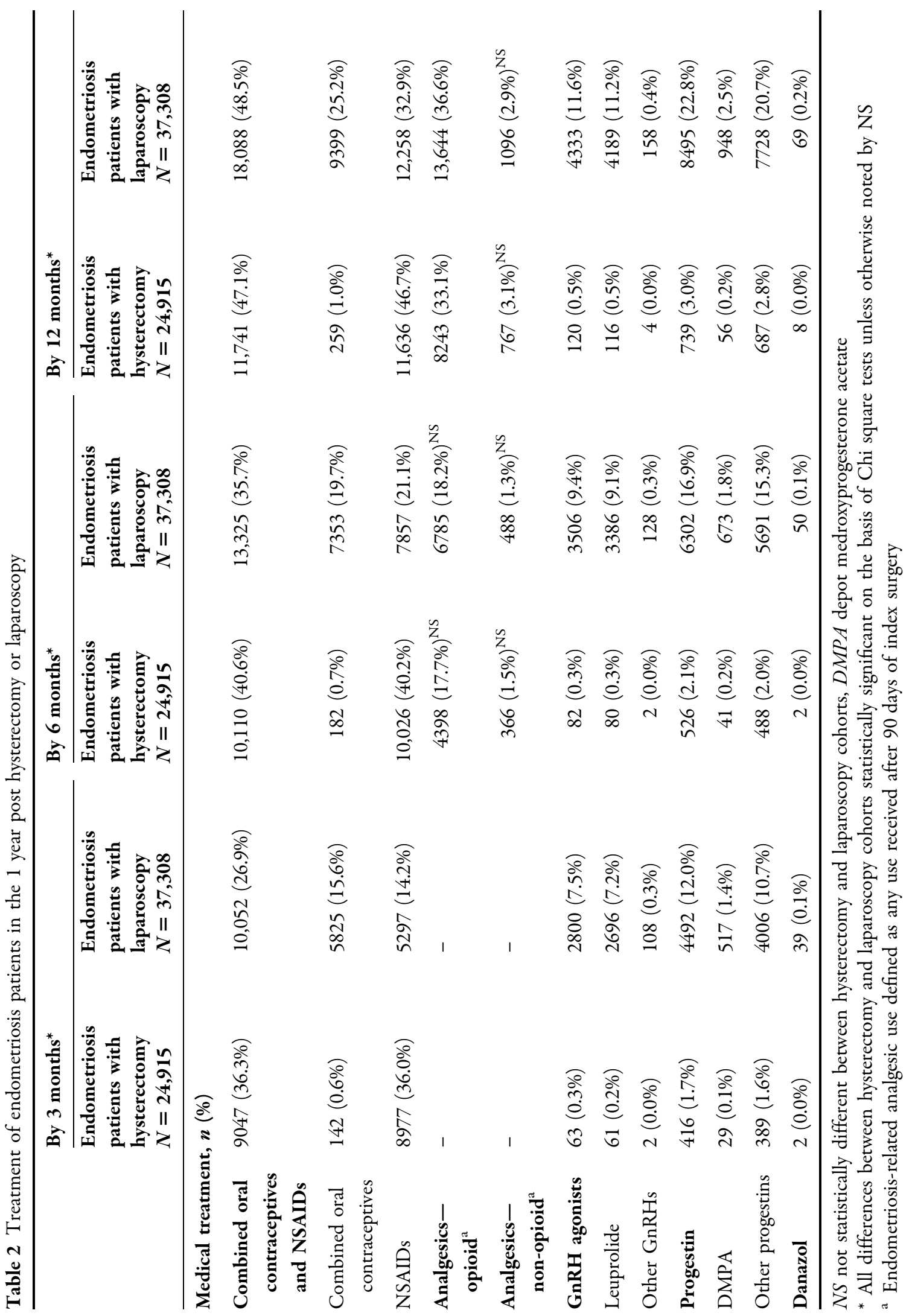


Table 3 Complications and endometriosis-related surgeries among endometriosis patients in the 1 year post hysterectomy or laparoscopy

\begin{tabular}{|c|c|c|}
\hline \multirow[t]{2}{*}{ Complications and endometriosis-related surgeries } & \multicolumn{2}{|l|}{ Endometriosis patients ${ }^{\phi}$} \\
\hline & With hysterectomy $N=24,915$ & With laparoscopy $N=37,308$ \\
\hline Complications $^{\mathrm{a}}, n(\%)$ & $11,457(46.0)$ & $13,590(36.4)$ \\
\hline Medical/surgical complications & $2590(10.4)$ & $1786(4.8)$ \\
\hline Venous thromboembolism & $206(0.8)$ & $181(0.5)$ \\
\hline Pulmonary embolus & $38(0.2)$ & $21(0.1)$ \\
\hline Thrombophlebitis & $181(0.7)$ & $167(0.4)$ \\
\hline Menopausal symptoms & $2624(10.5)$ & $1199(3.2)$ \\
\hline Gastritis and duodenitis & $848(3.4)^{\mathrm{NS}}$ & $1310(3.5)^{\mathrm{NS}}$ \\
\hline Nephropathy & $19(0.1)$ & $12(0.0)$ \\
\hline Infection & $8157(32.7)$ & $10,931(29.3)$ \\
\hline Wound infection ${ }^{\mathrm{b}}$ & $2834(11.4)$ & $3986(10.7)$ \\
\hline Urinary tract infection & $4850(19.5)$ & $6289(16.9)$ \\
\hline Abscess & $1134(4.6)^{\mathrm{NS}}$ & $1608(4.3)^{\mathrm{NS}}$ \\
\hline Pyelonephritis & $112(0.4)$ & $29(0.1)$ \\
\hline Cellulitis & $185(0.7)$ & $131(0.4)$ \\
\hline Sepsis & $108(0.4)$ & $69(0.2)$ \\
\hline Fever/pyrexia & $1336(5.4)$ & $1545(4.1)$ \\
\hline Vascular repair & $14(0.1)^{\mathrm{NS}}$ & $13(0.0)^{\mathrm{NS}}$ \\
\hline Damage to blood vessels & $1(0.0)^{\mathrm{NS}}$ & $0(0.0)^{\mathrm{NS}}$ \\
\hline Genitourinary tract injury & $617(2.5)$ & $514(1.4)$ \\
\hline Cystotomy & $2(0.0)^{\mathrm{NS}}$ & $2(0.0)^{\mathrm{NS}}$ \\
\hline Ureteral injury (transection) & $35(0.1)$ & $21(0.1)$ \\
\hline Vesicovaginal fistula & $65(0.3)$ & $15(0.0)$ \\
\hline Ureterovaginal fistula & $8(0.0)$ & $0(0.0)$ \\
\hline Urinary retention & $522(2.1)$ & $475(1.3)$ \\
\hline Bladder atony & $12(0.0)^{\mathrm{NS}}$ & $9(0.0)^{\mathrm{NS}}$ \\
\hline Gastrointestinal tract injury & $119(0.5)$ & $117(0.3)$ \\
\hline Injury to gastrointestinal tract & $36(0.1)^{\mathrm{NS}}$ & $34(0.1)^{\mathrm{NS}}$ \\
\hline Anastomotic dehiscence/anastomotic leak syndrome & $34(0.1)$ & $28(0.1)$ \\
\hline Rectovaginal fistula & $22(0.1)$ & $14(0.0)$ \\
\hline Rectal stenosis & $5(0.0)^{\mathrm{NS}}$ & $13(0.0)^{\mathrm{NS}}$ \\
\hline Rectal perforation & $31(0.1)^{\mathrm{NS}}$ & $39(0.1)^{\mathrm{NS}}$ \\
\hline
\end{tabular}


Table 3 continued

\begin{tabular}{|c|c|c|}
\hline \multirow[t]{2}{*}{ Complications and endometriosis-related surgeries } & \multicolumn{2}{|l|}{ Endometriosis patients ${ }^{\phi}$} \\
\hline & With hysterectomy $N=24,915$ & With laparoscopy $N=37,308$ \\
\hline Bleeding & $810(3.3)$ & $394(1.1)$ \\
\hline Blood transfusion & $49(0.2)$ & $30(0.1)$ \\
\hline Hematoma & $809(3.2)$ & $392(1.1)$ \\
\hline Vascular injury & $2(0.0)^{\mathrm{NS}}$ & $2(0.0)^{\mathrm{NS}}$ \\
\hline Vaginal cuff dehiscence & $229(0.9)$ & $140(0.4)$ \\
\hline Nerve injury & $173(0.7)$ & $157(0.4)$ \\
\hline Repair to bladder & $35(0.1)$ & $221(0.6)$ \\
\hline Repair to ureter & $70(0.3)$ & $61(0.2)$ \\
\hline Repair to bowel & $21(0.1)$ & $9(0.0)$ \\
\hline Other complications $^{c}$ & $104(0.4)$ & $67(0.2)$ \\
\hline
\end{tabular}

$N S$ not statistically different between hysterectomy and laparoscopy cohorts

$\phi$ All differences between hysterectomy and laparoscopy cohorts statistically significant $(P<0.05)$ on the basis of Chi square tests unless otherwise noted by NS

a Diagnosis and procedure codes used to identify complications are included in the supplementary material

b Wound infections included acute parametritis and pelvic cellulitis, chronic or unspecified parametritis and pelvic cellulitis, vaginitis, and vulvovaginitis, postoperative infection

c Other complications included accidental cut, puncture, perforation, or hemorrhage during medical care and surgical procedures as the cause of abnormal reaction of patient or later complication

was associated with greater odds of an all-cause (14.5\% vs $8.8 \%)$ or endometriosis-related $(4.5 \%$ vs $0.8 \%$ ) hospitalization ( $4.5 \%$ vs $0.8 \%$ ) after the index procedure, as well as significantly longer all-cause $(0.8$ vs 0.5 days; $P<0.0001)$ and endometriosis-related $\quad(0.2$ vs 0.0 days; $P<0.0001)$ LOS after the index procedure.

While Table 4 examines hospitalization rates in the year after the index procedure, Fig. 2 examines such rates for the first 14 days, 1 month, and 3 months post index event. In addition to reiterating the finding that hysterectomy patients were generally more likely to be hospitalized for endometriosis or for any cause in the first 90 days after the index date, Fig. 2 also shows that the rate of subsequent hospitalizations, for any cause and for endometriosis treatment, climbed steadily among the laparoscopy cohort over the first 3 months after the index surgery. Among the hysterectomy cohort, all-cause hospitalizations likewise became increasingly frequent over the first 3 months; however, the rate of endometriosis-related hospitalizations did not increase after the first 14 days. Among women who had a hysterectomy, almost half of all subsequent all-cause hospitalizations and 75\% of all endometriosis-related subsequent hospitalizations occurred in the first 90 days after the initial procedure. In contrast, for women whose initial procedure was a laparoscopy, less than $25 \%$ of subsequent all-cause or endometriosis-related hospitalizations occurred in the first 90 days after the initial procedure.

In addition to the primary results presented above, the analyses were also re-examined for patients who received a hysterectomy according to the type of procedure. Specifically, women who received a hysterectomy were subgrouped into abdominal $(N=7439)$, laparoscopic $(N=13,611)$, or vaginal (3863) hysterectomy groups, with two patients with unspecified type 


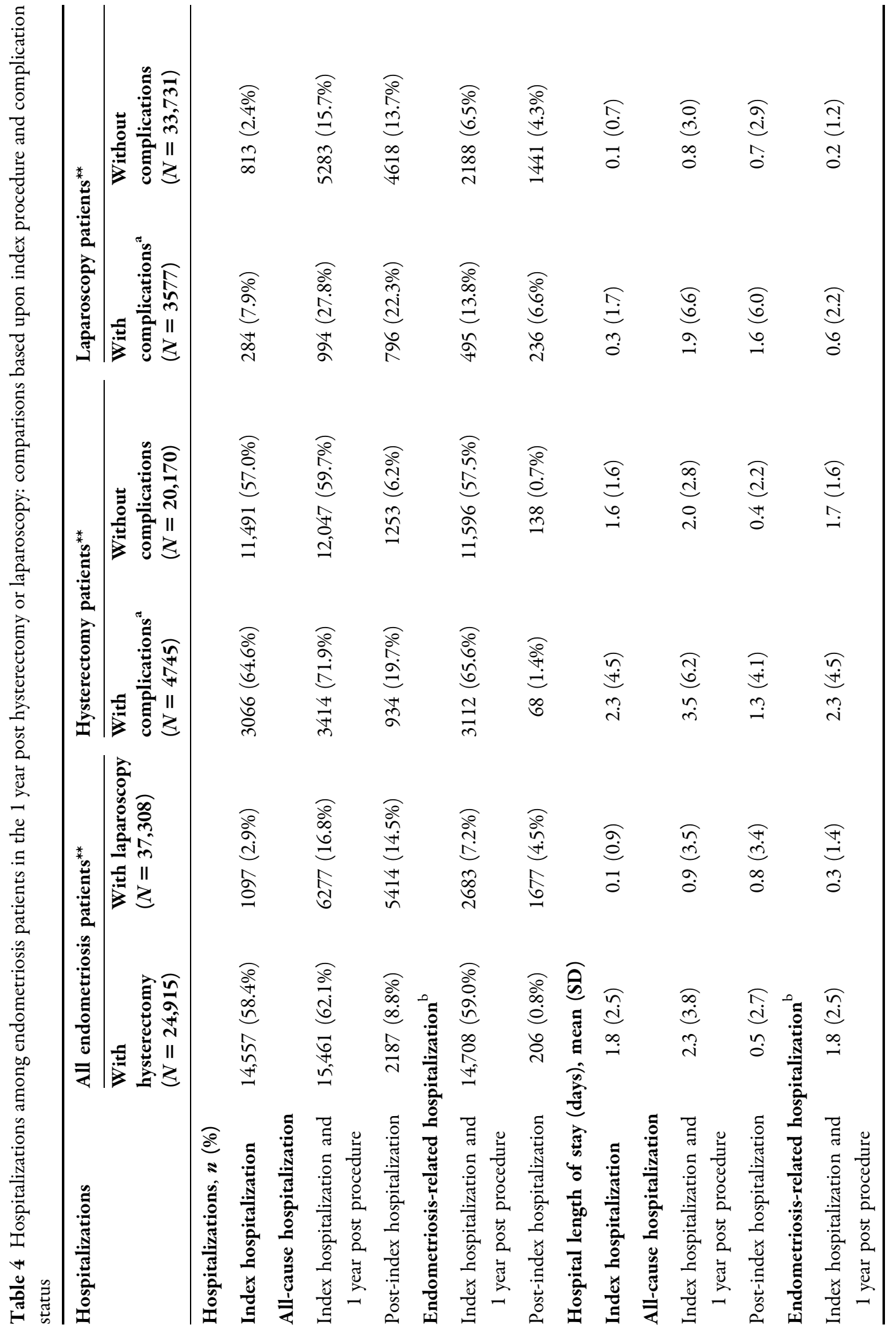




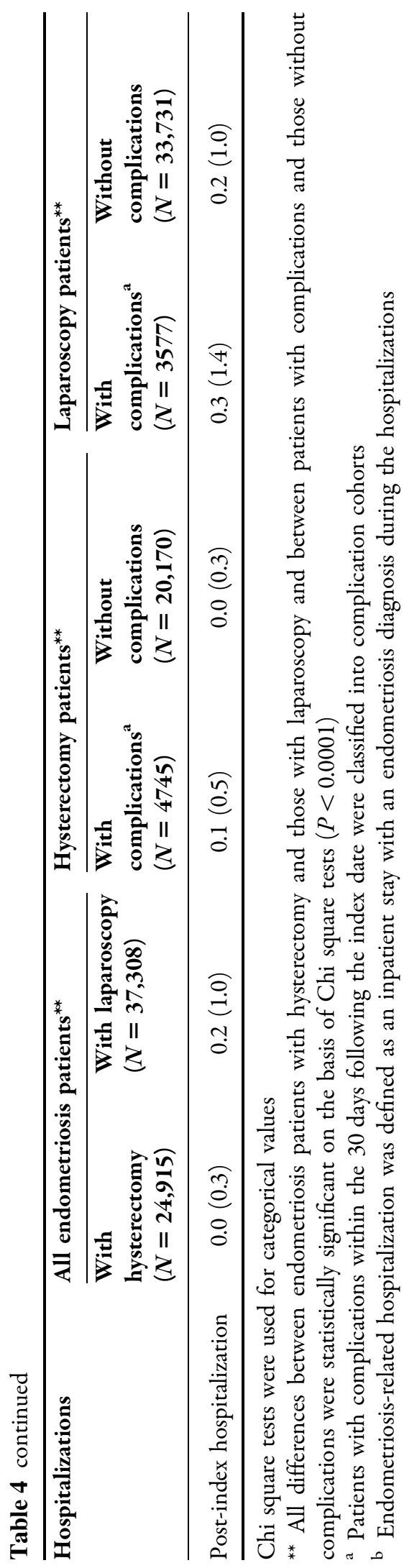

of hysterectomy not included in the analyses. Results, which are presented in Appendices S1-S5 in the supplementary material, illustrated that patients with abdominal hysterectomy are generally more likely to receive postsurgery treatments, develop complications, and have hospitalizations compared to patients who received either laparoscopic or vaginal hysterectomies. The trend in overall comparisons between hysterectomy and laparoscopy is still preserved when each hysterectomy subgroup is compared to the laparoscopy cohort.

\section{DISCUSSION}

Laparoscopy and hysterectomy are common treatments for endometriosis and the two most frequent surgical interventions for the disease [14]. In a previous, large, claims-based analysis, $31.6 \%$ of all endometriosis patients underwent a laparoscopy and 29\% a hysterectomy (primarily abdominal or, less frequently, vaginal) within the first year after their diagnosis, and both of these procedures were reported to be associated with significant direct costs [14]. Consistent with that research, both of these treatment types were found in the present study to be associated with a substantial burden of negative outcomes, including significant health care resource use. The following sections further discuss and compare the baseline profiles and outcomes of these two surgical cohorts, particularly within the context of previous literature.

\section{Baseline Characteristics}

Consistent with the fact that hysterectomies are contraindicated for women who wish to maintain their fertility [21], the women in the hysterectomy cohort were significantly older than women whose initial procedure was a laparoscopy ( 41.4 vs 35.4 years; $P<0.0001$ ). In addition, in agreement with research from as early as the 1970s [22] and as recent as 2003 [23], the hysterectomy patients were more likely to reside in the South and less likely to reside in the Northeast. 

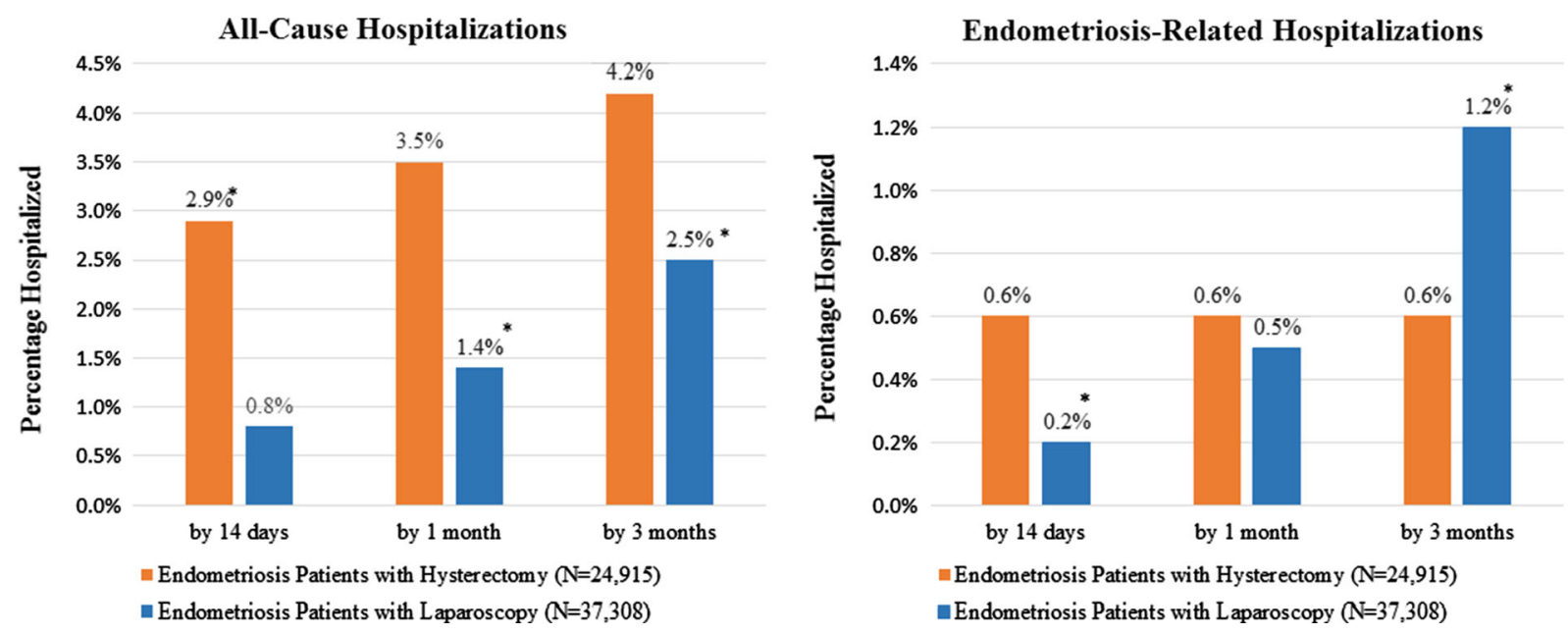

Fig. 2 Rates of all-cause and endometriosis-related hospitalizations over time. ${ }^{*} p<0.05$ based upon Chi square tests. Endometriosis patients with hysterectomy $-N=24,915$; Endometriosis patients with laparoscopy $-N=37,308$

\section{Postsurgical Outcome: Pain and Medication Use}

Consistent with previous evidence [13], prescription drug use was highly prevalent among both cohorts of patients during the first year after their index surgery. In agreement with research reporting that more invasive surgical procedures are associated with a higher likelihood of an opioid prescription [24], the prevalence of opioid use in the hysterectomy cohort rose sharply from $17.7 \%$ at 6 months post procedure to $33.1 \%$ at 1 year post procedure, while these rates increased from $18.2 \%$ to $36.6 \%$ in the laparoscopy cohort over the same time period. Note that use of these drugs was defined as any such prescriptions received 90 days after the index surgery to preclude short-term treatment of postoperative pain. The high prevalence of opioid prescriptions, as well as the sustained use of such drugs post procedure, is notable given previously reported associations between prescription opioid use for chronic, noncancer pain and opioids abuse [25] or overdose [26]. Relative to the hysterectomy cohort, the laparoscopy patients were more likely to have received hormonal therapies in the postsurgery period, consistent with previous literature indicating that the use of such therapies can lower the odds of recurrence and subsequent laparoscopies [1, 27, 28].
In contrast, the use of any hormone therapy was less frequent in the hysterectomy cohort compared to the laparoscopy cohort, congruent with previous evidence that hormone replacement therapy has little impact on outcomes [29] and increases the odds of pain recurrence [21] after an endometriosis-related hysterectomy.

\section{Postsurgical Outcomes: Complications and Subsequent Endometriosis-Related Surgeries}

The hysterectomy cohort in the present study had a very high rate of complications (at 46\%) relative to the $30 \%$ complication rate associated with hysterectomies overall in the USA [30]. This finding is consistent with the evidence that hysterectomies used to treat endometriosis can be highly complex and require surgeons with specialized skills, especially in the case of radical surgery which eliminates all possible endometriosis implants found in the pelvic and abdominal cavities [31, 32]. In addition, the high complication rate seen in this study is consistent with the findings that a majority of patients in the hysterectomy cohort were hospitalized for their procedure (58.4\%) and approximately one-third were prescribed an opioid to control pain at 1 year post procedure 
(33.1\%); in addition, nearly half $(46.7 \%)$ of the hysterectomy patients used a prescription NSAID in the 1 year after their initial hysterectomy. Congruent with previous research [33], infections were the most common type of surgical complication for the hysterectomy patients. After infections, the most frequent complication type was menopausal symptoms, a finding which is consistent with previous literature reporting that hysterectomy may result in early menopause, even when the ovaries are preserved [1]. A comparison across alternative type of hysterectomies (see Appendix S2) revealed that, in general, abdominal hysterectomy was associated with higher complication rates than laparoscopic or vaginal hysterectomy. However, patients who had an abdominal hysterectomy were significantly less likely to experience a genitourinary tract injury.

Consistent with earlier research [18], the laparoscopy patients had a greater rate of subsequent surgery relative to the hysterectomy cohort ( $14.5 \%$ vs $8.8 \%)$, and $5.6 \%$ of the laparoscopy patients went on to have a hysterectomy during the follow-up period. Previous studies have reported higher frequencies of follow-on laparoscopies (21-55\%) [13, 18] and subsequent hysterectomy (12\%) [34] among laparoscopy patients, in concert with the longer follow-up periods in those studies and the evidence that the frequency of subsequent surgeries increases over time after an initial laparoscopy $[13,18]$. In addition to the significant risk of a hospitalization associated with these follow-on operations (see Table 4 and Fig. 2), subsequent surgeries after a primary laparoscopy have been associated with substantially reduced fertility rates among women seeking to conceive [35]. The rate of repeat surgeries among the hysterectomy patients in the present study was $13.0 \%$, which is consistent with previous evidence of a $15 \%$ prevalence of persistent symptoms after a hysterectomy to treat endometriosis [29]. In previous studies, the long-term rates of reoperation after a hysterectomy were significantly higher $(23 \%$ or $31 \%)$ when one or both ovaries was preserved, but significantly lower $(8 \%$ or $10 \%)$ when the ovaries were removed $[18,36]$.

\section{Postsurgical Outcomes: Hospitalization Rates and LOS}

In agreement with the general categorization of laparoscopy as a minimally invasive surgery [37] with less associated morbidity and lower associated costs relative to more invasive procedures [8], the laparoscopy patients in this study had a lower rate of complications relative to the hysterectomy cohort $(36.4 \%$ vs $46.0 \%)$, and less than half the likelihood of a medical/surgical complication (4.8\% vs $10.4 \%)$. Also consistent with the minimally invasive nature of the surgery, relatively few (2.9\%) laparoscopy patients were hospitalized with the initial surgery, and the average LOS for a patient who was hospitalized was only roughly $2 \mathrm{~h}$ (0.10 days). However, the laparoscopy patients in this study had a higher percentage of complications relative to the $0.2-10.3 \%$ frequency of complications previously associated with most gynecologic laparoscopies [38], consistent with previous evidence that gynecologic laparoscopies involving extensive adhesion removal have the highest complication rates [39]. The complexity of such surgeries [39] and the high rate of infections suggest why $10 \%$ of the laparoscopy patients were rehospitalized for reasons other than endometriosis in the postsurgery period. In addition, the laparoscopy patients in this study were more likely relative to the hysterectomy patients to be subsequently hospitalized to treat endometriosis ( $4.5 \%$ vs $0.8 \%)$.

This study found a $14.5 \%$ rate of hospitalization at 1 year post laparoscopy, as well as an increasing rate of hospitalizations over the first 3 months of the postsurgical period (Fig. 2). These findings are generally consistent with previous research which found a $27 \%$ rate of rehospitalization within a 4-year follow-up period after an initial laparoscopy [34]. Figure 2 and Table 3 illustrate that if patients had an endometriosis-related hospitalization or surgery after their initial hysterectomy, this event typically occurred within the first 14 days and was followed by yet another hysterectomy or an oophorectomy. These findings suggest that debilitating symptoms returned almost immediately when parts of the uterus and/or the ovaries were preserved. However, consistent 
with previous evidence that a hysterectomy to treat endometriosis is definitive (i.e., with $0 \%$ pain recurrence) when it includes the removal of the uterus, adnexa, ovaries, fallopian tubes, and all accessible endometriotic lesions [31], Fig. 2 suggests that once more/all of the uterus and/or the ovaries were removed in the first 14 days post index surgery, there was no further endometriosis-related hospitalization within the first 3 months. The rate of all-cause hospitalizations among the hysterectomy patients continued to climb after the first 14 days, however, consistent with the high rate of surgical complications seen in the cohort.

\section{Limitations}

As with any research, the present findings should be evaluated within the context of the study's limitations. Since the records were obtained from commercially insured women aged 18-45 years residing in the USA, the results may not apply to all women with endometriosis or to other subpopulations. Second, the results are descriptive in nature, and do not control for potential reasons why alternative procedures are selected, such as selection bias, physician ability and comfort level, or the role of insurance compensation. The study also does not control for the potential placebo effect associated with surgical procedures in general and laparoscopy in particular. For example, research has shown that the placebo response for surgery is similar to the response for other therapeutic interventions, and accounts for up to $35 \%$ of patient response [40]. Similarly, an examination of laparoscopic procedures for endometriosis found that the surgery was associated with a $30 \%$ placebo effect, although the authors found that laparoscopy was more effective than placebo at reducing pain and improving quality of life [41].

In addition to the above limitations, the diagnoses and treatments under study were all identified on the basis of procedure codes and other diagnostic records, which may have been subject to coding errors or bias due to misdiagnoses or underdiagnoses. The reliance on such data also precluded any measure of disease severity or surgical techniques, both of which may affect the rate of disease recurrence. The data was also unable to distinguish between patients with and without oophorectomies, which may be associated with differences in patient outcomes. Finally, the 1-year follow-up period may have led to an underestimate of the burden associated with endometriosis, given that longer-term research has suggested that the rate of repeat surgery increases over time after a laparoscopy or a hysterectomy $[13,18]$.

\section{CONCLUSION}

The endometriosis patients in this analysis had a considerable risk of surgical complications, subsequent surgeries, and hospital admissions, both during and after their initial therapeutic laparoscopy or hysterectomy. Relative to the laparoscopy cohort, the hysterectomy patients had a greater risk of being hospitalized with their index surgery, and of being diagnosed with surgical complications and prescribed analgesics, including opioids, after their initial procedure. Relative to the hysterectomy patients, the laparoscopy cohort had a higher rate of subsequent surgeries and a greater risk of hospital admissions during the 1-year follow-up period. As compared to their baseline medication use, the laparoscopy patients also received more hormone therapy after the index surgery. The results of this study suggest that better nonsurgical treatments could potentially improve patient outcomes and the quality of life of millions of American women.

\section{ACKNOWLEDGEMENTS}

This study, the article processing charges, and open access fee were funded by AbbVie. AbbVie participated in data analysis, interpretation of data, review, and approval of the manuscript. All named authors meet the International Committee of Medical Journal Editors (ICMJE) criteria for authorship for this manuscript, take responsibility for the integrity of the work as a whole, and have given final approval for the version to be published. Editorial assistance in the preparation of this manuscript was provided 
by Dr. Maureen Lage and Dr. Michael Treglia of HealthMetrics Outcomes Research, LLC. Support for this assistance was funded by Abbvie, Inc.

Disclosures. Eric Surrey is medical director at the Colorado Center for Reproductive Medicine, has served in a consulting role on research to AbbVie, and is on the speaker bureau for Ferring Laboratories. Ahmed Soliman is an AbbVie employee and may hold AbbVie stocks or stock options. Bowdoin $\mathrm{Su}$ is an AbbVie employee and may hold AbbVie stocks or stock options. Hongbo Yang is an employee of Analysis Group, which received a research contract to conduct this study with and on behalf of AbbVie. Ella Du is an employee of Analysis Group, which received a research contract to conduct this study with and on behalf of AbbVie.

Compliance with Ethics Guidelines. This article does not contain any new studies with human or animal subjects performed by any of the authors.

Data Availability. The datasets generated and analyzed during the current study are not publicly available because of licensing issues, but are available from the corresponding author on reasonable request.

Open Access. This article is distributed under the terms of the Creative Commons Attribution-NonCommercial 4.0 International License (http://creativecommons.org/licenses/by-nc/4. $0 /$ ), which permits any noncommercial use, distribution, and reproduction in any medium, provided you give appropriate credit to the original author(s) and the source, provide a link to the Creative Commons license, and indicate if changes were made.

\section{REFERENCES}

1. Practice Committee of the American Society for Reproductive Medicine. Treatment of pelvic pain associated with endometriosis: a committee opinion. Fertil Steril. 2014;101(4):927-35.
2. Schleedoorn MJ, Nelen WLDM, Dunselman GA, Vermeulen N, EndoKey Group. Selection of key recommendations for the management of women with endometriosis by an international panel of patients and professionals. Hum Reprod. 2016;31(6):1208-18.

3. Dunselman GA, Vermeulen N, Becker C, et al. ESHRE guideline: management of women with endometriosis. Hum Reprod. 2014;29(3):400-12.

4. National Institutes of Health: Eunice Kennedy Shriver National Institute of Child Health and Human Development. How many people are affected by or at risk for endometriosis? 2013. https://www.nichd.nih.gov/health/topics/endometri/ conditioninfo/Pages/at-risk.aspx. Accessed cited 17 Apr 2017.

5. womenshealth.gov. Endometriosis| womenshealth.gov. 2014. http://www.womenshealth. gov/publications/our-publications/fact-sheet/endo metriosis.html. Accessed cited 17 Apr 2017.

6. Simoens S, Dunselman G, Dirksen C, et al. The burden of endometriosis: costs and quality of life of women with endometriosis and treated in referral centres. Hum Reprod. 2012;27(5):1292-9.

7. Soliman AM, Yang H, Du EX, Kelley C, Winkel C. The direct and indirect costs associated with endometriosis: a systematic literature review. Hum Reprod. 2016;31(4):712-22.

8. Gao X, Outley J, Botteman M, Spalding J, Simon JA, Pashos CL. Economic burden of endometriosis. Fertil Steril. 2006;86(6):1561-72.

9. National Institutes of Health: Eunice Kennedy Shriver National Institute of Child Health and Human Development. What are the treatments for endometriosis? 2013. https://www.nichd.nih.gov/ health/topics/endometri/conditioninfo/Pages/treat ment.aspx\#pain. Accessed cited 17 Apr 2017.

10. Schrager, Sarina, Falleroni, Julianne, Edgoose, Jennifer. Evaluation and treatment of endometriosisAmerican family physician. American Family Physician. 2013. http://www.aafp.org/afp/2013/ 0115/p107.html. Accessed cited 17 Apr 2017.

11. Surrey ES. Endometriosis and assisted reproductive technologies: maximizing outcomes. Semin Reprod Med. 2013;31(2):154-63.

12. The American College of Obstetricians and Gynecologists. Endometriosis-ACOG. www.acog.org. 2012. http://www.acog.org/Patients/FAQs/ Endometriosis. Accessed cited 17 Apr 2017.

13. Vercellini P, Crosignani PG, Abbiati A, Somigliana E, Viganò P, Fedele L. The effect of surgery for 
symptomatic endometriosis: the other side of the story. Hum Reprod Update. 2009;15(2):177-88.

14. Fuldeore M, Chwalisz K, Marx S, et al. Surgical procedures and their cost estimates among women with newly diagnosed endometriosis: a US database study. J Med Econ. 2011;14(1):115-23.

15. Garry R, Clayton R, Hawe J. The effect of endometriosis and its radical laparoscopic excision on quality of life indicators. BJOG Int J Obstet Gynaecol. 2000;107(1):44-54.

16. Giudice LC. Clinical practice. Endometriosis. N Engl J Med. 2010;362(25):2389-98.

17. Koga K, Takemura Y, Osuga Y, et al. Recurrence of ovarian endometrioma after laparoscopic excision. Hum Reprod. 2006;21(8):2171-4.

18. Shakiba K, Bena JF, McGill KM, Minger J, Falcone T. Surgical treatment of endometriosis: a 7-year follow-up on the requirement for further surgery. Obstet Gynecol. 2008;111(6):1285-92.

19. Parker WH, Broder MS, Liu Z, Shoupe D, Farquhar $\mathrm{C}$, Berek JS. Ovarian conservation at the time of hysterectomy for benign disease. Obstet Gynecol. 2005;106(2):219-26.

20. Soliman A, Du E, Yang H, Wu J, Castelli-Haley J. Retreatment rates among endometriosis patients undergoing hysterectomy or laparoscopy. J Womens Health. 2017;26(6):644-54.

21. Rizk B, Fischer AS, Lotfy HA, et al. Recurrence of endometriosis after hysterectomy. Facts Views Vis Obgyn. 2014;6(4):219-27.

22. Walker AM, Jick H. Temporal and regional variation in hysterectomy rates in the United States, 1970-1975. Am J Epidemiol. 1979;110(1):41-6.

23. Wu JM, Wechter ME, Geller EJ, Nguyen TV, Visco AG. Hysterectomy rates in the United States, 2003. Obstet Gynecol. 2007;110(5):1091-5.

24. Clarke H, Soneji N, Ko DT, Yun L, Wijeysundera DN. Rates and risk factors for prolonged opioid use after major surgery: population based cohort study. BMJ. 2014;11(348):g1251.

25. Reid MC, Engles-Horton LL, Weber MB, Kerns RD, Rogers EL, O'Connor PG. Use of opioid medications for chronic noncancer pain syndromes in primary care. J Gen Intern Med. 2002;17(3):173-9.

26. Dunn KM, Sanders KW, Rutter CM, et al. Opioid prescriptions for chronic pain and overdose: a cohort study. Overdose and prescribed opioids: associations among chronic non-cancer pain patients. Ann Intern Med. 2010;152(2):85-92.
27. Garry R. The effectiveness of laparoscopic excision of endometriosis: Current Opinion in Obstetrics and Gynecology. LWW. 2004. http://journals.lww. com/co-obgyn/Fulltext/2004/08000/The_effective ness_of_laparoscopic_excision_of.5.aspx. Accessed cited 17 Apr 2017.

28. Soliman AM, Bonafede M, Farr AM, Castelli-Haley J, Winkel C. Analysis of subsequent surgery rates among endometriosis patients who underwent surgery with and without concomitant leuprolide acetate therapy. Curr Med Res Opin. 2016;32(6):1073-82.

29. Vercellini P, Barbara G, Abbiati A, Somigliana E, Viganò P, Fedele L. Repetitive surgery for recurrent symptomatic endometriosis: what to do? Eur J Obstet Gynecol Reprod Biol. 2009;146(1):15-21.

30. National Women's Health Network. Hysterectomy. NWHN. $2015 . \quad$ https://www.nwhn.org/ hysterectomy/. Accessed cited 26 Apr 2016.

31. Fedele L, Bianchi S, Zanconato G, Berlanda N, Borruto F, Frontino G. Tailoring radicality in demolitive surgery for deeply infiltrating endometriosis. Am J Obstet Gynecol. 2005;193(1):114-7.

32. De la Hera-Lazaro CM, Munoz-Gonzalez JL, Perez RO, et al. Radical surgery for endometriosis: analysis of quality of life and surgical procedure. Clin Med Insights Womens Health. 2016;9:7-11.

33. Clarke-Pearson DL, Geller EJ. Complications of hysterectomy. Obstet Gynecol. 2013;121(3):654-73.

34. Weir E, Mustard C, Cohen M, Kung R. Endometriosis: what is the risk of hospital admission, readmission, and major surgical intervention? J Minim Invasive Gynecol. 2005;12(6):486-93.

35. Berlanda N, Vercellini P, Fedele L. The outcomes of repeat surgery for recurrent symptomatic endometriosis. Curr Opin Obstet Gynecol. 2010;22(4):320-5.

36. Namnoum AB, Hickman TN, Goodman SB, Gehlbach DL, Rock JA. Incidence of symptom recurrence after hysterectomy for endometriosis. Fertil Steril. 1995;64(5):898-902.

37. The American College of Obstetricians and Gynecologists. Laparoscopy-ACOG. 2015. http://www. acog.org/Patients/FAQs/Laparoscopy. Accessed cited 31 Aug 2016.

38. Magrina JF. Complications of laparoscopic surgery. Clin Obstet Gynecol. 2002;45(2):469-80.

39. Querleu D, Chapron C, Chevallier L, Bruhat MA. Complications of gynecologic laparoscopic 
surgery-a French multicenter collaborative study. N Engl J Med. 1993;328(18):1355.

40. Wolf BR, Buckwalter JA. Randomized surgical trials and "sham" surgery: relevance to modern orthopaedics and minimally invasive surgery. Iowa Orthop J. 2006;26:107-11.
41. Abbott J, Hawe J, Hunter D, Holmes M, Finn P, Garry R. Laparoscopic excision of endometriosis: a randomized, placebo-controlled trial. Fertil Steril. 2004;82(4):878-84. 